\title{
ON A METRIC THAT GHARACTERIZES DIMENSION
}

\section{J. DE GROOT}

1. Introduction. Sometimes it is possible to characterize topological properties of a metrizable space $M$ by claiming that a certain (topologypreserving) metric $\rho$ can be introduced in $M$. For example:

( $\alpha$ ) A metrizable space $C$ is compact, that is, is a compactum, if and only if $C$ is totally bounded ${ }^{1}$ in every metric.

( $\beta$ ) A metrizable space $M$ is separable, if and only if there exists a totally bounded metric in $M$.

( $\gamma$ ) A (non-empty) metrizable space $M$ is 0 -dimensional ( $\operatorname{dim} M=0$ ), if and only if there exists a metric $\rho$ in $M$ which satisfies-instead of the triangle axiom - the stronger axiom

$$
\rho(y, z) \leqslant \max [\rho(x, y), \rho(x, z)],
$$

(that is, every "triangle" in this metric has two equal "sides" and the third "side" is smaller than or equal to the other ones) (see 2, 3).

Nagata (7) gave a characterization of a metrizable space $M$ of $\operatorname{dim} \leqslant n$ (for every non-negative integer $n$ ) by means of a certain metric, which he showed to be equivalent with $(\gamma)$ in the case $n=0$. However, this characterization (see \$2) is rather complicated. In this note we give another generalization of $(\gamma)$ which gives a simplification of Nagata's result for arbitrary dimension $n$, but only for the case of separable metrizable spaces, i.e., metrizable spaces with a countable base.

Theorem. A topological space $M$ is a separable metrizable space of dimension $\leqslant n$ if and only if one can introduce a totally bounded metric $\rho$ in $M$ satisfying the following condition: for every $n+3$ points

$$
x, y_{1}, y_{2}, y_{3}, \ldots y_{k}, \ldots y_{n+2}
$$

in $M$ there is a triplet of indices $i, j, k$, such that

$$
\rho\left(y_{i}, y_{j}\right) \leqslant \rho\left(x, y_{k}\right),
$$

Corollary. A compactum has dimension $\leqslant n$, if and only if one can introduce a metric $\rho$, such that for every $n+3$ points $x, y_{k}(k=1,2, \ldots, n+2)$ the relation 1.2 holds for suitable $i, j, k$.

\footnotetext{
Received May 28, 1957.
}

${ }^{1} \epsilon$-net: A finite number of points $p$ such that the system of $\epsilon$-neighbourhoods cover the space. Totally bounded: there is an $\epsilon$-net for every $\epsilon>0$. See (1) in general for our terminology. See (4) for dimension theory in separable metrizable spaces and $(\mathbf{5} ; \mathbf{6})$ for dimension theory in metrizable spaces. 
It has to be observed that condition 1.2 is essentially weaker than the condition which is satisfied by Nagata's metric (7) (see also $\S 2$ ). Indeed, the ordinary metric of a segment of real numbers is a metric $\rho$ with 1.2 (for the case $n=2$ ), but does not satisfy Nagata's condition.

2. Proof of Theorem. Suppose $M$ is a separable metric space with $\operatorname{dim} M \leqslant n$. Since $M$ is separable, we can embed $M$, according to a theorem of Hurewicz, in a compactum $\bar{M}$, such that $M$ is dense in $\bar{M}$, and

$$
\operatorname{dim} M=\operatorname{dim} \bar{M} \leqslant n .
$$

We introduce in $\bar{M}$ the metric $\rho$ of Nagata (7), which has the following characterizing property: for every $\epsilon>0$ and for every point $\mathrm{x} \in \bar{M}$ the relations ${ }^{2}$

$2.1 \quad \rho\left(U_{\frac{1}{2} \epsilon}(x), y_{k}\right)<\epsilon \quad(k=1,2, \ldots n+2)$,

where $U_{\delta}(x)$ is the set of all points $p$ with $\rho(x, p)<\delta$, imply

$$
\min _{i \neq j} \rho\left(y_{i}, y_{j}\right)<\epsilon \text {. }
$$

It is easy to see that this metric $\rho$ in particular satisfies our condition 1.2. Indeed, being given the points $x, y_{k}(k=1,2, \ldots n+2)$, consider all $\epsilon$ with

$$
\epsilon>\mu=\max _{k} \rho\left(x, y_{k}\right) .
$$

For these $\epsilon, 2.1$ obviously holds, so 2.2 holds.

Since inf $\epsilon=\mu$, we have

$$
\min _{i \neq j} \rho\left(y_{i}, y_{j}\right) \leqslant \mu \quad \text { q.e.d. }
$$

Moreover, the metric $\rho$ in the compact space $\bar{M}$ is necessarily totally bounded. Hence the metric $\rho$ of $M \subset \bar{M}$ is also totally bounded and satisfies 1.2 , which we had to prove.

Conversely, let $M$ have a totally bounded metric satisfying 1.2. $M$ is clearly separable. We shall now prove that $\operatorname{dim} M \leqslant n$.

$M$ can be extended, just as every metric space, to a complete metric space $\bar{M}$ in which $M$ is dense. Every sequence in $M$ has a Cauchy sequence (fundamental sequence) as subsequence, since $M$ is totally bounded under $\rho$. This Cauchy sequence converges in the complete $\bar{M}$. Hence $\bar{M}$ is compact and totally bounded under $\rho$, where $\rho$ now denotes the natural extension of $\rho$ (on $M)$ to $\bar{M}$. Property 1.2 also holds in this extended metric $\rho$ on $\bar{M}$. Indeed, suppose it does not hold for a set of certain points $\bar{x}, \bar{y}_{k}$. Then, since the distance function is continuous, we can determine small neighbourhoods of these points such that 1.2 does not hold for any set of points $x, y_{k}$ chosen in these neighbourhoods respectively. We can, however, choose these points $x, y_{k}$ from $M$, which leads to a coniradiction. We shall now prove $\operatorname{dim} \bar{M} \leqslant n$, from which follows $\operatorname{dim} M \leqslant n$.

${ }^{2}$ The distance of the sets $A$ and $B$ is denoted by $\rho(A, B)$. 
Consider an arbitrary finite open covering of $\bar{M}$. We have to find-according to the Lebesgue definition of dimension-a refinement of this covering of order $\leqslant n$ (i.e. each point of the refined covering is contained in at most $n+1$ elements of it).

Let $\sigma=2 \epsilon$ be a Lebesgue number of the given finite covering of $\bar{M}$. Choose a maximal set $p_{1}, p_{2}, \ldots, p_{s}$ in $\bar{M}$ such that $\rho\left(p_{i}, p_{j}\right) \geqslant \epsilon$ for all $i, j$ with $i \neq j$. This set of points $\left\{p_{i}\right\}$ is an $\epsilon$-net of $\bar{M}$ and the covering

$(i=1,2, \ldots s)$

is a refinement of the given covering. If a point $x \in \bar{M}$ belongs to at least $n+2$ elements of 2.3 , we have $\rho\left(x, p_{i}\right)<\epsilon$ for $n+2$ different points $p_{i}$. Hence, using $1.2, \rho\left(p_{i}, p_{j}\right)<\epsilon$ for suitable $i, j$ with $i \neq j$, which is contradictory to the definition of $\left\{p_{i}\right\}$. Hence, the order of 2.3 is $\leqslant n$, so $\operatorname{dim} \bar{M} \leqslant n$.

3. Questions. The corollary admits an immediate generalization to semicompact $^{3}$ metrizable spaces, since we can apply in this case the sum theorem of dimension theory (a metric space which is the countable sum of closed subsets of dimension $\leqslant n$, has dimension $\leqslant n$ ), while the proof in the other direction is covered by Nagata's theorem, as mentioned in $\$ 2$. So, our characterization by means of a metric satisfying 1.2 includes for example $n$-dimensional Euclidean spaces as well.

However, it remains uncertain whether in separable metric spaces $M$ the property $\operatorname{dim} \leqslant n$ can be characterized by a metric satisfying 1.2 only. There might be a possibility that the condition of total boundedness can be omitted in this case, if the condition 1.2 is strengthened in the following way: there is a metric $\rho$ in $M$ which satisfies 1.2 and also, if $\rho\left(x, y_{1}\right)=\rho\left(x, y_{2}\right)=\ldots$ $=\rho\left(x, y_{n+2}\right)$,

$$
\rho\left(y_{i}, y_{j}\right)<\rho\left(x, y_{k}\right), \text { for suitable } i, j, k
$$$$
(i \neq j) \text {. }
$$

However, does there exist such a metric? For $n=0$, the answer is in the affirmative $(4, \S 2)$.

The problem of generalizing the Theorem to metric spaces in general remains unanswered too.

${ }^{3} \mathrm{~A}$ space is semicompact if it is the sum of a countable number of compact spaces. Every locally compact, separable, metrizable space is semicompact, since such a space can be compactified by one point. 


\section{REFERENCES}

1. P. Alexandrov and H. Hopf, Topologie (Berlin 1935).

2. J. de Groot and H. de Vries, $A$ note on non-archimedean metrizations, Indagationes Math., 17 (1955), 222-224.

3. J. de Groot, Non-archimedian metrics in topology, Proc. Amer. Math. Soc., 7 (1956), 948953.

4. W. Hurewicz, H. Wallman, Dimension Theory (Princeton 1941).

5. M. Katětov, On the dimension of non-separable spaces $I$, Tsjechoslov. Mat. Zj., 2 (77) (1952), 333-368.

6. K. Morita, Normal families and dimension theory for metric spaces, Math. Ann., 128 (1954), $350-362$.

7. J. Nagata, On a relation between dimension and metrization, Proc. Jap. Ac., 32 (1956), 237240.

Mathematisch Instituut

University of Amsterdam 